This item was submitted to Loughborough's Research Repository by the author.

Items in Figshare are protected by copyright, with all rights reserved, unless otherwise indicated.

\title{
Output-feedback shared-control for fully actuated linear mechanical systems
}

PLEASE CITE THE PUBLISHED VERSION

https://doi.org/10.1109/acc.2014.6859061

PUBLISHER

IEEE @ AACC

VERSION

AM (Accepted Manuscript)

LICENCE

CC BY-NC-ND 4.0

REPOSITORY RECORD

Jiang, Jingjing, and Alessandro Astolfi. 2019. "Output-feedback Shared-control for Fully Actuated Linear Mechanical Systems”. figshare. https://hdl.handle.net/2134/36760. 


\title{
Output-Feedback Shared-Control for Fully Actuated Linear Mechanical Systems
}

\author{
Jingjing Jiang $^{1}$ and Alessandro Astolfi ${ }^{2}$
}

\begin{abstract}
This paper presents an output feedback sharedcontrol algorithm for fully-actuated, linear, mechanical systems. The feasible configurations of the system are described by a group of linear inequalities which characterize a convex admissible set. The properties of the shared-control algorithm are established with a Lyapunov-like analysis. Simple numerical examples demonstrate the effectiveness of the strategy.
\end{abstract}

\section{INTRODUCTION}

Shared-control, as the name implies, is an algorithm to unite more than one control signals. For simplicity, in this paper, we consider two inputs, an external human input $u_{h}$ and an output-feedback control input $u_{o f}$. The human operator is in charge of the system most of the time and $u_{o f}$ is active only in emergency cases, for example when the system evolves towards "dangerous" situations (to be formally defined). Shared-control is of great importances because a large number of systems involve human operators. These systems integrate the adaptive, interactive and inventive task execution skills of human beings and the reliable, precise, inexhaustible and fast task execution capability of automated controllers.

The technique of human-in-the-loop has been significantly developed in the last two decades and it is still an active research area. Typical applications of shared-control are medical devices [1], mobility assistance [2] [3], tele-operation [4] [5], driving [6] [7], robotics [8], training systems [9] [10] and transportation systems [11] [12]. A shared-control strategy is used in [13] to help a disabled person to safely operate a wheelchair. By setting the level of support the operator can achieve obstacle avoidance, smooth speed and turn around functions.

A vivid metaphor of shared-control is horse riding [14]: "When people loose the rein, the horse would run automatically while by slightly increasing the force on the rein, the control authority is shifted from the horse to the human being smoothly."

A method to combine the human operator's input and the feedback control input through a hierarchical structure has

${ }^{1}$ J. Jiang is with the Dept. of Electrical and Electronic Engineering, Imperial College London, SW7 2AZ, UK, E-mail: jingjing. jiang10@imperial.ac.uk

${ }^{2} \mathrm{~A}$. Astolfi is with the Dept. of Electrical and Electronic Engineering, Imperial College London, London, SW7 2AZ, UK and DICII, University of Roma "Tor Vergata", Via del Politecnico 1, 00133 Rome, Italy, E-mail: a.astolfieimperial.ac.uk been given in [15], while [16] has presented a Lyapunov-like solution of the shared-control problem.

As stated at the beginning of this section our shared-control is designed mainly for safety issues, among which obstacle avoidance is the most common one. Obstacle avoidance problems have been solved in various ways. The Vector Field Histogram method is an often used way to solve the problem for mobile robots [17]. This method suffers from various limitations, similarly to the Virtual Force Field method [18], such as the occurrence of local minima and the insurgence of oscillations while passing through narrow areas [18]. Fuzzy control is another commonly used method. For example, [19] gives a group of fuzzy control rules combing "negative" rules (obstacle avoidance) and "positive" rules (directing the robot to the target). The system controlled by these fuzzy rules suffers from stability problems: no theoretical proof of stability can be given.

Shared control problems have been studied in [20], where a sharing rule based on a discrete event framework has been given, and in [21] where a continuous scalar function to guarantee the smooth transition from the human input to the control input has been designed. A scheme to distribute control authority among several inputs by introducing an augmented signal based on input's magnitude and rate saturation levels has been constructed in [22]. In [23], the shared-control problem has been studied using some of the ideas of [22] and [24] for fully-actuated, linear, mechanical systems for which the whole state is measurable. In this paper we extend the results in [23] to the case in which only the generalized positions of the mechanical system are measurable.

The rest of the paper is organized as follows. In Section II, the shared-control problem is formulated. Section III introduces a solution to the problem followed by several formal properties of the controlled system. The shared-control algorithm is illustrated via two simple examples and simulation results are given in Section IV. Finally, Section V gives some conclusions and discusses future work.

\section{PROBLEM STATEMENT}

In this section we formulate the shared-control problem for fully-actuated, linear, mechanical systems by partial state feedback.

Consider a fully-actuated, linear, mechanical system, the 
dynamics of which are described by the equation

$$
\mathcal{M} \ddot{p}+\mathcal{K} \dot{p}+\mathcal{G} p=u_{s},
$$

where $p(t) \in \mathbb{R}^{n}$ describes the generalized positions, $u_{s}(t) \in$ $\mathbb{R}^{n}$ is the control input, $\mathcal{M}=\mathcal{M}^{\prime}>0$ denotes the (constant) inertia matrix, $\mathcal{K}=\mathcal{K}^{\prime} \geq 0$ is the (constant) Coulumb friction coefficient and $\mathcal{G}=\mathcal{G}^{\prime}$ models the potential force.

Suppose the output of the system (1) is given by the equation

$$
y=C\left[\begin{array}{l}
p \\
\dot{p}
\end{array}\right]
$$

and assume the system (1)-(2) is observable. Let $\hat{p}$ and $\hat{v}$ denote the estimates of $p$ and $\dot{p}$, respectively and define the estimation error as $e(t)=[p(t)-\hat{p}(t), v(t)-\hat{v}(t)]^{T}$.

The estimates are obtained from measurements of $y$ and $u_{s}$, via a dynamical system of the form

$$
\left[\begin{array}{c}
\dot{\hat{p}} \\
\hat{\hat{v}}
\end{array}\right]=\mathcal{A}\left[\begin{array}{c}
\hat{p} \\
\hat{v}
\end{array}\right]+\mathcal{B} u_{s}+\mathcal{H} y
$$

The shared-control input $u_{s}$ is defined as

$$
u_{s}(\hat{p}, \hat{v}, t)=[1-k(\hat{p}, \hat{v})] u_{o f}(\hat{p}, \hat{v}, t)+k(\hat{p}, \hat{v}) u_{h}(\hat{p}, \hat{v}, t),
$$

where $u_{o f}$ denotes the output-feedback control input, $u_{h}$ denotes the operator's input and $k \in[0,1]$ quantifies how the input is shared between $u_{o f}$ and $u_{h}$.

Similarly to [23], we use the name s-control to denote the shared-control action, the name $h$-control to denote the human action, the name $f$-control to denote the outputfeedback control action, and the name sharing function to denote the function $k$. In addition, we use the name s-closedloop to represent the system described by (1), (2), (3) and (4), and the name $h$-closed-loop to represent the system described by the equations (2), (3) and

$$
\mathcal{M} \ddot{p}+\mathcal{K} \dot{p}+\mathcal{G} p=u_{h} .
$$

The h-closed-loop and the s-closed-loop share the same state space, namely $\mathcal{P} \times \mathcal{V} \times \hat{\mathcal{P}} \times \hat{\mathcal{V}}=\mathbb{R}^{n} \times \mathbb{R}^{n} \times \mathbb{R}^{n} \times \mathbb{R}^{n}$ where $\mathcal{P}$ denotes the configuration set, $\hat{\mathcal{P}}=\mathcal{P}, \mathcal{V}$ denotes the velocity set, and $\hat{\mathcal{V}}=\mathcal{V}$. Let $\mathcal{P}_{a} \subset \mathcal{P}$ be a closed, compact set and assume it is the set of admissible configurations. Then the output-feedback shared-control problem can be stated as follows.

Given a system (1)-(2) with an h-control $u_{h}(\hat{p}, \hat{v}, t)$ and an admissible configuration set $\mathcal{P}_{a}$, find (if possible)

- matrices $\mathcal{A}, \mathcal{B}$ and $\mathcal{H}$;

- a f-control $u_{o f}$;

- a sharing function $k$;

- a safe set $\mathcal{R}_{s}(t): \mathcal{R}_{s}(t) \triangleq \hat{\mathcal{P}}_{s} \times \hat{\mathcal{V}}_{s} \subseteq \mathcal{P}_{a} \times \hat{\mathcal{V}}_{s} \subset$ $\mathcal{P}_{a} \times \hat{\mathcal{V}} \triangleq \mathcal{R}$ and such that ${ }^{1} \lim _{t \rightarrow \infty} \mathcal{R}_{s}(t)=\mathcal{P}_{a} \times \hat{\mathcal{V}}_{s}$;

if

${ }^{1}$ The limit of a set $\mathcal{S}(t)$, denoted as $\lim _{t \rightarrow \infty} \mathcal{S}(t)$, equals to $\mathcal{Q}$ if and only

$$
\forall \epsilon>0, \exists t_{\epsilon}>0: \mathcal{Q}^{\epsilon-} \subset \mathcal{S}(t) \subset \mathcal{Q}^{\epsilon+} \text { for all } t \geq t_{\epsilon},
$$

such that the following properties of the s-closed-loop system hold.

(P0) The estimation error system

$$
\begin{aligned}
\dot{e} & =\left(\left[\begin{array}{cc}
0 & I \\
-\mathcal{M}^{-1} \mathcal{G} & -\mathcal{M}^{-1} \mathcal{K}
\end{array}\right]-\mathcal{H C}\right) e \\
& +\left(\left[\begin{array}{c}
0 \\
\mathcal{M}^{-1}
\end{array}\right]-\mathcal{B}\right) u_{s}-\mathcal{H C}\left[\begin{array}{c}
\hat{p} \\
\hat{v}
\end{array}\right] \\
& -\left(\mathcal{A}-\left[\begin{array}{cc}
0 & I \\
-\mathcal{M}^{-1} \mathcal{G} & -\mathcal{M}^{-1} \mathcal{K}
\end{array}\right]\right)\left[\begin{array}{l}
\hat{p} \\
\hat{v}
\end{array}\right]
\end{aligned}
$$

has an equilibrium at $e=0$ which is exponentially stable.

(P1) The set $\mathcal{R}$ is forward invariant.

(P2) Let $\Omega_{s}\left(\Omega_{h}\right.$, resp.) be the $\Omega$-limit set of the s-closedloop (h-closed-loop, resp.). Then

$$
\Omega_{s}= \begin{cases}\Omega_{h} & \text { if } \Omega_{h} \subset \mathcal{R}_{s}, \\ \Pi_{\mathcal{R}_{s}}\left(\Omega_{h}\right) & \text { if } \Omega_{h} \not \subset \mathcal{R}_{s},\end{cases}
$$

where $\Pi_{\mathcal{R}_{s}}\left(\Omega_{h}\right)$ is a projection of $\Omega_{h}$ into $\mathcal{R}_{s}$, which will be defined in Section III-A.

(P3) $u_{s}=u_{h}$ if $(\hat{p}, \hat{v}) \in \mathcal{R}_{s}$.

\section{DESIGN OF THE OUTPUT-FEEDBACK S-CONTROL}

In this section a shared-control strategy to solve the problem stated in Section II in the case in which $\mathcal{P}_{a}$ is described by a group of linear inequalities is given. For simplicity, we assume that only position sensors are available, which means $p(t)$ is measurable but $\dot{p}(t)$ is not.

The state-space representation of system (1) is given by

$$
\left\{\begin{array}{l}
\dot{p}=v, \\
\dot{v}=\mathcal{M}^{-1}\left(-\mathcal{K} v-\mathcal{G} p+u_{s}\right),
\end{array}\right.
$$

with output ${ }^{2}$

$$
y=\left[\begin{array}{ll}
I & 0
\end{array}\right]\left[\begin{array}{l}
p \\
v
\end{array}\right] .
$$

Without loss of generality, select

$$
\begin{aligned}
\mathcal{A} & =\left[\begin{array}{cc}
0 & I \\
-\mathcal{M}^{-1} \mathcal{G} & -\mathcal{M}^{-1} \mathcal{K}
\end{array}\right]-\mathcal{H}\left[\begin{array}{ll}
I & 0
\end{array}\right], \\
\mathcal{B} & =\left[\begin{array}{c}
0 \\
\mathcal{M}^{-1}
\end{array}\right],
\end{aligned}
$$

with $\mathcal{H}$ such that $\lambda(\mathcal{A}) \subset \mathbb{C}^{-}$. As a result (P0) holds and the signal $e(t)$ is exponentially converging to zero, i.e.

$$
\exists \alpha>0, \beta>0:\left|e_{i}(t)\right| \leq \mathscr{E}, \forall i \in\{1, \ldots, 2 n\},
$$

where $\mathcal{Q}^{\epsilon+}=\left\{B_{\epsilon}(x), x \in Q\right\}, \mathcal{Q}^{\epsilon-}=\overline{\left((\overline{\mathcal{Q}})^{\epsilon+}\right)}, B_{\epsilon}(x)$ is the ball centered at $x$ with radius $\epsilon>0$ and $\overline{\mathcal{Q}}$ denotes the complement of the set $\mathcal{Q}$.

${ }^{2} I$ and 0 denote the identity and zero matrix of appropriate dimensions. 
where $\mathscr{E}=\alpha e^{-\beta t} \max _{1 \leq i \leq 2 n}\left|e_{i}(0)\right|$.

Similarly to [23], assume $\mathcal{P}_{a}$ is defined by a group of linear inequalities, namely

$$
\mathcal{P}_{a}=\left\{p \in \mathbb{R}^{n} \mid \mathcal{S} p+\mathcal{T} \leq 0\right\},
$$

where $\mathcal{S}=\left[s_{1}^{T}, s_{2}^{T}, \ldots, s_{m}^{T}\right]^{T} \in \mathbb{R}^{m \times n}$ and $\mathcal{T}=$ $\left[t_{1}, t_{2}, \ldots, t_{m}\right]^{T} \in \mathbb{R}^{m}$.

Assumption 1: If $m>n$ then the matrices $\mathcal{S}$ and $\mathcal{T}$ satisfy the condition

$$
\operatorname{rank}\left(\left[\begin{array}{l}
s_{r_{1}} \\
\vdots \\
s_{r_{l}}
\end{array}\right]\right)<\operatorname{rank}\left(\left[\begin{array}{ll}
s_{r_{1}} & t_{r_{1}} \\
\vdots & \vdots \\
s_{r_{l}} & t_{r_{l}}
\end{array}\right]\right),
$$

for all $l \in[n+1, m]$ and $r_{1}, r_{2}, \ldots, r_{l} \in\{1,2, \ldots, m\}$.

In what follows we assume that Assumption 1 holds and $\mathcal{P}_{a}$ is not empty.

\section{A. Design of the f-control}

Without loss of generality, as detailed in [23], we design the f-control in the case $m=n$. Note also that, as discussed again in [23], $N_{c}$ f-controls, with $N_{c} \leq\left(\begin{array}{c}m \\ n\end{array}\right)$ have to be designed. Consider the $i^{\text {th }}$ group of constraints which is described by

$$
\mathcal{S}^{i} p+\mathcal{T}^{i} \leq 0
$$

where $\mathcal{S}^{i}=\left[s_{1}^{i^{T}}, s_{2}^{i^{T}}, \ldots, s_{n}^{i^{T}}\right]^{T}$ and $\mathcal{T}=\left[t_{1}^{i}, t_{2}^{i}, \ldots, t_{n}^{i}\right]^{T}$.

Define the new coordinate $x^{i}$ as $^{3}$

$$
x^{i}=\mathcal{S}^{i} \hat{p}+\mathcal{T}^{i}+\mathscr{E}\left|\mathcal{S}^{i} a\right|,
$$

where $a=[1,1, \ldots, 1]^{T}$ and

$$
\begin{aligned}
p_{r}^{i} & =\mathcal{S}^{i-1}\left(x_{r}^{i}-\mathcal{T}^{i}-\mathscr{E}\left|\mathcal{S}^{i} a\right|\right), \\
v_{r}^{i} & =\mathcal{S}^{i-1}\left(\dot{x}_{r}^{i}+\beta \mathscr{E}\left|\mathcal{S}^{i} a\right|\right) .
\end{aligned}
$$

From (8), we know that $x^{i} \leq 0$. However, this constraint is stronger than the original constraint (8) and it can be removed by changing the coordinate $x^{i}$ to $z^{i}=\left[z_{1}^{i}, z_{2}^{i}, \ldots, z_{n}^{i}\right]^{T}$ with

$$
z_{j}^{i}=\log \frac{x_{j}^{i}+\epsilon_{j}^{i}}{x_{r_{j}}^{i}+\epsilon_{r_{j}}^{i}}, \forall j \in\{1,2, \ldots, n\},
$$

where $x_{r_{j}}^{i}$ (to be defined) describes the desired evolution of the coordinate $x_{j}^{i}$ and

$$
\epsilon_{j}^{i}=\left\{\begin{array}{ll}
0, & \text { if } x_{j}^{i}<0, \\
<0, & \text { otherwise }
\end{array} \quad \epsilon_{r_{j}}^{i}= \begin{cases}0, & \text { if } x_{r_{j}}^{i}<0 \\
<0, & \text { otherwise }\end{cases}\right.
$$

The reference signal $x_{r}^{i}$, related to $x^{i}$, is given by

$$
x_{r}^{i}=\left[x_{r_{1}}^{i}, x_{r_{2}}^{i}, \ldots, x_{r_{n}}^{i}\right]^{T}
$$

${ }^{3}$ For a vector $b=\left[b_{1}, b_{2}, \ldots, b_{n}\right]^{T} \in \mathbb{R}^{n},|b|=\left[\left|b_{1}\right|,\left|b_{2}\right|, \ldots,\left|b_{n}\right|\right]^{T}$. with $x_{r_{j}}^{i}$ defined by

$$
x_{r_{j}}^{i}(t)= \begin{cases}0, & \text { if } h_{j}^{i}(t) \geq 0, \\ h_{j}^{i}\left(1-e^{\gamma h_{j}^{i}}\right), & \text { if } h_{j}^{i}(t)<0,\end{cases}
$$

where $\gamma>0, h_{j}^{i}(t)=s_{j}^{i} p_{d}(t)+t_{j}^{i}+\mathscr{E}(t)\left|s_{j}^{i} a\right|$ and $p_{d}$ denotes the reference configuration in the space $\mathcal{P}$. Note that $x_{r_{j}}^{i}$ is a non-positive smooth signal for all $j \in\{1,2, \ldots, n\}$. In addition, by (9), $\left(p_{r}^{i}, v_{r}^{i}\right) \in \mathcal{P}_{a} \times \mathbb{R}^{n}$.

Let $\left(p_{d}, \dot{p}_{d}\right)$ be a point of $\Omega_{h}$ in the $(p, v)$ space. The projection of $\left(p_{d}, \dot{p}_{d}\right)$ into $\mathcal{R}_{s}$ related to the $i^{\text {th }}$ group of constraints, denoted as $\Pi_{\mathcal{R}_{s}}^{i}\left(p_{d}, \dot{p}_{d}\right)$, is defined as

$$
\Pi_{\mathcal{R}_{s}}^{i}\left(p_{d}, \dot{p}_{d}\right)=\left(p_{r}^{i}, v_{r}^{i}\right)
$$

where $p_{r}^{i}$ and $v_{r}^{i}$ are given by equations (11) and (9). Then the projection of $\Omega_{h}$ into $\mathcal{R}_{s}$ related to the $i^{\text {th }}$ group of constraints is defined as

$$
\Pi_{\mathcal{R}_{s}}^{i}\left(\Omega_{h}\right)=\left\{s \in \mathcal{R}_{s} \mid s=\Pi_{\mathcal{R}_{s}}^{i}\left(p_{d}, \dot{p}_{d}\right), \forall\left(p_{d}, \dot{p}_{d}\right) \in \Omega_{h}\right\} .
$$

Finally, given $p_{r}^{i}$ and $v_{r}^{i}$, the reference input is calculated as

$$
u_{r}^{i}=\mathcal{M} \dot{v}_{r}^{i}+\mathcal{K} v_{r}^{i}+\mathcal{G} p_{r}^{i} .
$$

Using the variable $z^{i}$, the f-closed-loop system can be written as

$$
\begin{aligned}
\dot{z}^{i}= & \operatorname{diag}\left(\frac{e^{-z_{1}^{i}}-1}{x_{r_{1}}^{i}+\epsilon_{r_{1}}^{i}}, \ldots, \frac{e^{-z_{n}^{i}}-1}{x_{r_{n}}^{i}+\epsilon_{r_{n}}^{i}}\right)\left(\mathcal{S}^{i} v_{r}^{i}-\beta \mathscr{E}\left|\mathcal{S}^{i} a\right|\right) \\
& +\operatorname{diag}\left(\frac{e^{-z_{1}^{i}}}{x_{r_{1}}^{i}+\epsilon_{r_{1}}^{i}}, \ldots, \frac{e^{z_{n}^{i}}}{x_{r_{n}}^{i}+\epsilon_{r_{n}}^{i}}\right) \mathcal{S}^{i} \hat{v}_{e}^{i}, \\
\dot{\hat{v}}_{e}^{i}= & \mathcal{M}^{-1}\left(-\mathcal{K} \hat{v}_{e}^{i}-\mathcal{G} \hat{p}_{e}^{i}+u_{o f}^{i}-u_{r}^{i}\right),
\end{aligned}
$$

where

$$
\hat{p}_{e}^{i}=\left(\mathcal{S}^{i}\right)^{-1}\left[\begin{array}{c}
\left(x_{r_{1}}^{i}+\epsilon_{r_{1}}^{i}\right) e^{z_{1}^{i}}-\epsilon_{1}^{i}-x_{r_{1}}^{i}- \\
\ldots \\
\left(x_{r_{n}}^{i}+\epsilon_{r_{n}}^{i}\right) e^{z_{n}^{i}}-\epsilon_{n}^{i}-x_{r_{n}}^{i}
\end{array}\right] .
$$

Let $\hat{v}_{e}^{i *}=\left(\hat{v}_{e_{1}}^{i *}, \ldots, \hat{v}_{e_{n}}^{i *}\right)^{T}$ be the solution of the equations $\mathcal{S}^{i} \hat{v}_{e}^{i *}=z^{i}+\operatorname{diag}\left(e^{z_{1}^{i}}-1, \ldots, e^{z_{n}^{i}}-1\right)\left(\mathcal{S}^{i} v_{r}^{i}-\beta \mathscr{E}\left|\mathcal{S}^{i} a\right|\right)$.

Consider the Lyapunov function, associated to the $i^{\text {th }}$ group of constraints,

$$
L^{i}\left(z^{i}, \hat{v}_{e}^{i}\right)=\frac{1}{2}\left[z^{i^{T}} z^{i}+\left(\hat{v}_{e}^{i}-\hat{v}_{e}^{i *}\right)^{T}\left(\hat{v}_{e}^{i}-\hat{v}_{e}^{i *}\right)\right],
$$

and choose $u_{d}^{i}$ such that $L^{i}\left(z^{i}, \hat{v}_{e}^{i}\right)<0$ for all $z^{i} \neq 0$ and $\hat{v}_{e}^{i} \neq \hat{v}_{e}^{i *}$. One such a choice is given by

$$
\begin{aligned}
u_{o f}^{i}= & \mathcal{M}\left(\dot{\hat{v}}_{e}^{i *}-\mathcal{S}^{{ }^{T}}\left[\frac{z_{1}^{i} e^{-z_{1}^{i}}}{x_{r_{1}}^{i}+\epsilon_{r_{1}}^{i}}, \ldots, \frac{z_{n}^{i} e^{-z_{n}^{i}}}{x_{r_{n}}^{i}+\epsilon_{r_{n}}^{i}}\right]^{T}\right) \\
& -\eta \mathcal{M}\left(\hat{v}_{e}^{i}-\hat{v}_{e}^{i *}\right)+\mathcal{K} \hat{v}_{e}^{i}+\mathcal{G} \hat{p}_{e}{ }^{i}
\end{aligned}
$$

with $\eta>0$. 
Lemma 1: Consider the f-closed-loop (5)-(13) with $u_{s}=$ $u_{o f}^{i}$ given by (15), $u_{r}^{i}$ given by (12) and $x_{r}^{i}$ given by (11). Assume $p(0) \in \mathcal{P}_{a}$ and $\mathcal{S}^{i} \hat{p}(0)+\mathcal{T}^{i}+\mathscr{E}(0)\left|\mathcal{S}^{i} a\right| \leq 0$ for all $i \in\left[1, N_{c}\right]$. Then the system has the following properties:

$$
\begin{aligned}
& \lim _{t \rightarrow \infty}\left(p(t)-p_{r}(t)\right)=0, \text { where } p_{r}(t) \text { is given by (9); } \\
& p(t) \in \mathcal{P}_{a}, \text { for all } t \geq 0 .
\end{aligned}
$$

\section{B. Shared-control Algorithm}

As stated in Section II the safe set $\mathcal{R}_{s}(t)$ needs to be defined before designing the sharing function $k$. With reference to the $i^{\text {th }}$ group of constraints, three subsets, the safe set $\mathcal{R}_{s}^{i}(t)$, the hysteresis set $\mathcal{R}_{h}^{i}(t)$ and the dangerous set $\mathcal{R}_{d}^{i}(t)$, are defined in equations (16) (top of the next page), where ${ }^{4} \mathcal{X}_{a}^{i}=$ $\mathcal{S}^{i} \mathcal{P}_{a}+\mathcal{T}^{i}+\mathscr{E}\left|\mathcal{S}^{i} a\right|$ and $b_{2}>b_{1}>0$.

To eliminate confusions on the set definitions for different groups of constraints, it is important to pull the subsets given in (16) back into the $(\hat{p}, \hat{v})$ coordinates. This can be done using the equations

$$
\begin{aligned}
\overline{\mathcal{R}}_{s}^{i}= & \operatorname{diag}\left(\mathcal{S}^{\mathrm{i}-1}, \mathcal{S}^{\mathrm{i}-1}\right)\left(\mathcal{R}_{\mathrm{s}}^{\mathrm{i}}-\operatorname{col}\left(\mathcal{T}^{\mathrm{i}}, 0_{\mathrm{n}}\right)+\right. \\
& \left.\operatorname{diag}\left(\mathcal{S}^{\mathrm{i}-1}, \mathcal{S}^{\mathrm{i}-1}\right) \operatorname{col}(-\mathscr{E} \mathrm{a}, \beta \mathscr{E} \mathrm{a})\right), \\
\overline{\mathcal{R}}_{h}^{i}= & \operatorname{diag}\left(\mathcal{S}^{\mathrm{i}-1}, \mathcal{S}^{\mathrm{i}-1}\right)\left(\mathcal{R}_{\mathrm{h}}^{\mathrm{i}}-\operatorname{col}\left(\mathcal{T}^{\mathrm{i}}, 0_{\mathrm{n}}\right)+\right. \\
& \left.\operatorname{diag}\left(\mathcal{S}^{\mathrm{i}-1}, \mathcal{S}^{\mathrm{i}-1}\right) \operatorname{col}(-\mathscr{E} \mathrm{a}, \beta \mathscr{E} \mathrm{a})\right), \\
\overline{\mathcal{R}}_{d}^{i}= & \operatorname{diag}\left(\mathcal{S}^{\mathrm{i}-1}, \mathcal{S}^{\mathrm{i}-1}\right)\left(\mathcal{R}_{\mathrm{d}}^{\mathrm{i}}-\operatorname{col}\left(\mathcal{T}^{\mathrm{i}}, 0_{\mathrm{n}}\right)+\right. \\
& \left.\operatorname{diag}\left(\mathcal{S}^{\mathrm{i}-1}, \mathcal{S}^{\mathrm{i}-1}\right) \operatorname{col}(-\mathscr{E} \mathrm{a}, \beta \mathscr{E} \mathrm{a})\right),
\end{aligned}
$$

where $\operatorname{col}(a, b)$ is a column vector obtained by stacking the vector $b$ under $a$. By construction the sets $\overline{\mathcal{R}}_{s}^{i}, \overline{\mathcal{R}}_{h}^{i}$ and $\overline{\mathcal{R}}_{d}^{i}$, have the following properties:

- $\overline{\mathcal{R}}_{s}^{i} \cup \overline{\mathcal{R}}_{h}^{i} \cup \overline{\mathcal{R}}_{d}^{i} \subseteq \mathcal{R}$, and $\lim _{t \rightarrow \infty}\left(\overline{\mathcal{R}}_{s}^{i} \cup \overline{\mathcal{R}}_{h}^{i} \cup \overline{\mathcal{R}}_{d}^{i}\right)=$ $\mathcal{R}, \forall i \in\left\{1,2, \ldots, N_{c}\right\}$

- $\overline{\mathcal{R}}_{s}^{i} \cap \overline{\mathcal{R}}_{d}^{i}=\left\{(\hat{p}, \hat{v})\left|\mathcal{S}^{i} \hat{p}+\mathcal{T}^{i}+\mathscr{E}\right| \mathcal{S}^{i} a \mid=0, \hat{v}=0\right\}, \forall i \in$ $\left\{1,2, \ldots, N_{c}\right\}$;

- $\overline{\mathcal{R}}_{d}^{i} \cap \overline{\mathcal{R}}_{d}^{j}=\emptyset, \forall i \neq j$ and $i, j \in\left\{1,2, \ldots, N_{c}\right\}$.

Similarly to [23], the sharing function, based on the three subsets $\overline{\mathcal{R}}_{s}^{i}, \overline{\mathcal{R}}_{h}^{i}$ and $\overline{\mathcal{R}}_{d}^{i}$, is defined as

$$
k_{i}(\hat{p}, \hat{v})= \begin{cases}1, & (\hat{p}, \hat{v}) \in \overline{\mathcal{R}}_{s}^{i} \backslash \overline{\mathcal{R}}_{d}^{i} \\ l_{i}(\hat{p}, \hat{v}), & (\hat{p}, \hat{v}) \in \overline{\mathcal{R}}_{h}^{i} \\ 0, & (\hat{p}, \hat{v}) \in \overline{\mathcal{R}}_{d}^{i}\end{cases}
$$

where

$$
l_{i}(\hat{p}, \hat{v})= \begin{cases}1, & \text { if }(\hat{p}, \hat{v}) \text { enters } \overline{\mathcal{R}}_{h}^{i} \text { from } \overline{\mathcal{R}}_{s}^{i} \\ 0, & \text { if }(\hat{p}, \hat{v}) \text { enters } \overline{\mathcal{R}}_{h}^{i} \text { from } \overline{\mathcal{R}}_{d}^{i}\end{cases}
$$

\footnotetext{
${ }^{4}$ The set $\mathcal{S}^{i} \mathcal{P}_{a}+\mathcal{T}^{i}+\mathscr{E}\left|\mathcal{S}^{i} a\right|$, with $\mathcal{S}^{i} \in \mathbb{R}^{n \times n}, \mathcal{T}^{i} \in \mathbb{R}^{n}, a=$ $[1,1, \ldots, 1]^{T}$ and $\mathcal{P}_{a} \in \mathbb{R}^{n}$ is defined as

$\mathcal{S}^{i} \mathcal{P}_{a}+\mathcal{T}^{i}+\mathscr{E}\left|\mathcal{S}^{i} a\right|=\left\{x \in \mathbb{R}^{n}\left|x=S^{i} y+T^{i}+\mathscr{E}\right| \mathcal{S}^{i} a \mid, y \in \mathcal{P}_{a}\right\}$.
}

Note that the set $\mathcal{X}_{a}^{i}$ is a time-dependent set, since $\mathscr{E}$ is a function of time.
The f-controller relative to the $i^{\text {th }}$ group of constraints, given by $(15)$, can be rewritten in the $(\hat{p}, \hat{v})$ coordinates as

$$
\begin{aligned}
& u_{o f}^{i}=-\eta \mathcal{M}\left(\hat{v}-v_{r}^{i}-\hat{v}_{e}^{i *}\right)+\mathcal{K}\left(\hat{v}-v_{r}^{i}\right)+\mathcal{G}\left(\hat{p}-p_{r}^{i}\right) \\
& \mathcal{M}\left(\dot{\hat{v}}_{e}^{i *}-\mathcal{S}^{i T}\left[\frac{\log \frac{m_{1}^{i}}{r_{1}^{i}}}{m_{1}^{i}}, \ldots, \frac{\log \frac{m_{n}^{i}}{r_{n}^{i}}}{m_{n}^{i}}\right]^{T}\right),
\end{aligned}
$$

where $\hat{v}_{e}^{i *}=\gamma \mathscr{E} a+\mathcal{S}^{i-1}\left[\frac{\log \frac{m_{1}^{i}}{r_{1}^{i}}}{m_{1}^{i}}, \ldots, \frac{\log \frac{m_{n}^{i}}{r_{n}^{i}}}{m_{n}^{i}}\right]^{T}+$ $\operatorname{diag}\left(\frac{\mathrm{m}_{1}^{\mathrm{i}}}{\mathrm{r}_{1}^{\mathrm{i}}}-1, \ldots, \frac{\mathrm{m}_{\mathrm{n}}^{\mathrm{i}}}{\mathrm{r}_{\mathrm{n}}^{\mathrm{i}}}-1\right) \mathrm{v}_{\mathrm{r}}^{\mathrm{i}}, m_{j}^{i}=s_{j}^{i} \hat{p}+t_{j}^{i}+\mathscr{E}\left|s_{j}^{i} a\right|+\epsilon_{j}^{i}$ and $r_{j}^{i}=s_{j}^{i} p_{r}^{i}+t_{j}^{i}+\epsilon_{r_{j}}^{i}$ for all $j \in\{1,2, \ldots, n\}$.

Finally, the s-control input can be written in the $(\hat{p}, \hat{v})$ coordinates as

$u_{s}(\hat{p}, \hat{v})=\min _{1 \leq i \leq N_{c}} k_{i}(\hat{p}, \hat{v}) u_{h}+\sum_{i=1}^{N_{c}}\left[\left(1-k_{i}(\hat{p}, \hat{v})\right) u_{o f}^{i}(\hat{p}, \hat{v})\right]$.

Note that, as in [23], for each time instant the condition $k^{i}(\hat{p}, \hat{v})=0$ is satisfied for at most one $i \in\left\{1,2, \ldots, N_{c}\right\}$.

Theorem 1: Consider the system (5)-(6) with the sharedcontrol action (17), (18) and (19). Assume that $p(0) \in \mathcal{P}_{a}$, $\mathcal{S}^{i} \hat{p}(0)+\mathcal{T}^{i}+\mathscr{E}(0)\left|\mathcal{S}^{i} a\right| \leq 0$ for all $i \in\left[1, N_{c}\right]$ and $\Omega_{h}$ is feasible. Then there exist $\eta>0$ and $b_{2}>b_{1}>0$ such that the s-closed-loop has the following properties.

(1) $p(t) \in \mathcal{P}_{a}$ for all $t \geq 0$.

(2) $\Omega_{s}=\Omega_{h}$.

(3) $u_{s}(t)=u_{h}(t)$ for all $t \geq 0$ and $(\hat{p}(t), \hat{v}(t)) \in \mathcal{R}_{s} \backslash \mathcal{R}_{d}$.

Remark 1: The shared-control algorithm presented above can also be applied to noncovex admissible regions defined via linear inequalities and logical "statements". The only difference is the way in which the three subsets $\mathcal{R}_{s}, \mathcal{R}_{h}$ and $\mathcal{R}_{d}$ are defined. In fact, as shown in [25], any concave polygons can be partitioned by $c$ lines (virtual bounds) into $d$ convex polygons, where $c$ and $d$ are positive integers. Hence, any concave polygons can be defined by $d$ groups of convex polygons complemented with logic statements (related to the virtual bounds). Let ${ }^{i} \mathcal{R}_{s},{ }^{i} \mathcal{R}_{h}$ and ${ }^{i} \mathcal{R}_{d}$ denote the three subsets related to the $i^{\text {th }}$ convex sub-polygon. Then the overall safe, hysteresis and dangerous subsets are defined as

$$
\begin{aligned}
& \mathcal{R}_{s}={ }^{1} \mathcal{R}_{s} \cup{ }^{2} \mathcal{R}_{s} \cup \cdots \cup{ }^{d} \mathcal{R}_{s}, \\
& \mathcal{R}_{h}={ }^{1} \mathcal{R}_{h} \cup{ }^{2} \mathcal{R}_{h} \cup \cdots \cup{ }^{d} \mathcal{R}_{h}, \\
& \mathcal{R}_{d}={ }^{1} \mathcal{R}_{d} \cup{ }^{2} \mathcal{R}_{d} \cup \cdots \cup{ }^{d} \mathcal{R}_{d},
\end{aligned}
$$

and we could apply the same construction described for the case of a convex admissible region. 


$$
\begin{gathered}
\mathcal{R}_{s}^{i}=\left\{\left(x^{i}, \dot{x}^{i}\right) \in \mathcal{X}_{a}^{i} \times \mathbb{R}^{n}: \dot{x}_{j}^{i} \leq \frac{1}{x_{j}^{i}+b_{2}}-\frac{1}{b_{2}} \text { if } x_{j}^{i} \geq-b_{2} \text { for all } j \in\{1,2, \ldots, n\}\right\} \\
\mathcal{R}_{h}^{i}=\left\{\begin{array}{l}
\left.\left(x^{i}, \dot{x}^{i}\right) \in \mathcal{X}_{a}^{i} \times \mathbb{R}^{n}: \exists j \in\{1,2, \ldots, n\} \text { such that } \dot{x}_{j}^{i}>\frac{1}{x_{j}^{i}+b_{2}}-\frac{1}{b_{2}} \text { and } x_{j}^{i} \geq-b_{2}\right\} \\
\text { and } \dot{x}_{k}^{i}<\frac{1}{x_{k}^{i}+b_{1}}-\frac{1}{b_{1}} \text { if } x_{k}^{i} \geq-b_{1} \text { for all } k \in\{1,2, \ldots, n\}
\end{array}\right\} \\
\mathcal{R}_{d}^{i}=\left\{\begin{array}{l}
\left(x^{i}, \dot{x}^{i}\right) \in \mathcal{X}_{a}^{i} \times \mathbb{R}^{n}: \exists j \in\{1,2, \ldots, n\} \text { such that } \dot{x}_{j}^{i} \geq \frac{1}{x_{j}^{i}+b_{1}}-\frac{1}{b_{1}} \&-b_{1} \leq x_{j}^{i}<0 \\
\text { or } \exists j \in\{1,2, \ldots, n\} \text { such that } \dot{x}_{j}^{i}>\frac{1}{x_{j}^{i}+b_{1}}-\frac{1}{b_{1}} \text { and } x_{j}^{i}=0 \\
\text { or } \forall j \in\{1,2, \ldots, n\} \text { such that } x_{j}^{i}=\dot{x}_{j}^{i}=0
\end{array}\right\}
\end{gathered}
$$

\section{NUMERICAL EXAMPLES}

In this section we consider a fully actuated, linear, mechanical system with two degrees-of-freedom described by

$$
\begin{aligned}
& \dot{p}_{1}=v_{1}, \\
& \dot{p}_{2}=v_{2}, \\
& \dot{v}_{1}=-p_{1}-0.5 p_{2}-v_{1}-0.3 v_{2}+u_{1}, \\
& \dot{v}_{2}=-0.4 p_{1}-2 p_{2}-0.3 v_{1}+0.5 v_{2}+u_{2},
\end{aligned}
$$

and two admissible sets: a convex one and a concave one. The target trajectories in both cases are infeasible.

\section{A. Convex $\mathcal{P}_{a}$}

Consider the system (20) with the admissible set described by

$$
\mathcal{P}_{a}=\left\{p=\left[p_{1}, p_{2}\right]^{T} \mid p_{1} \geq 0 \text { and } p_{2} \geq 0\right\} .
$$

Assume the output $p$ of the system has to track the trajectory

$$
p_{d}(t)=[1.7-0.1 t, 1.8-0.1 t]^{T} .
$$

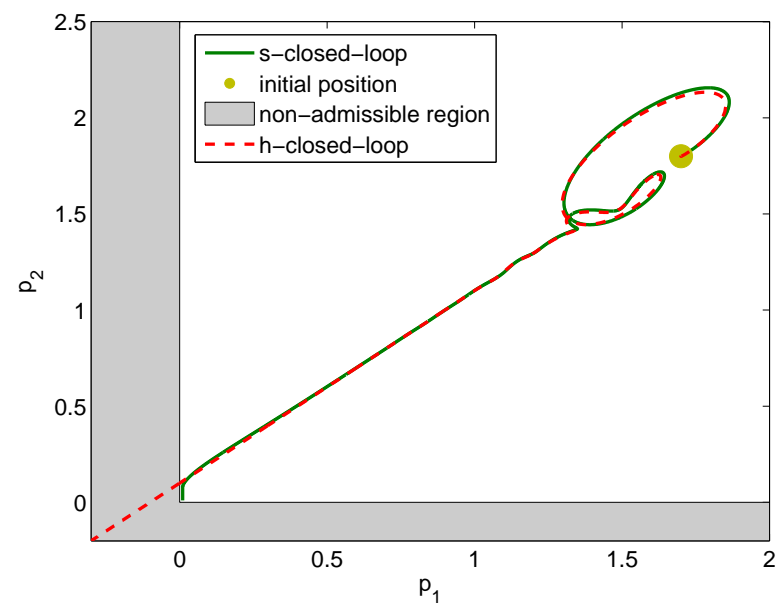

Fig. 1. Trajectories of the system (20) for the set $\mathcal{P}_{a}$ given in (21): hclosed-loop (red, dashed) and s-closed-loop (green, solid).
Figure 1 shows that the output trajectory of the s-closedloop system coincides with that of the h-closed-loop system for small values of $t$. This is because the trajectory is far away from the boundaries of the admissible set. However, the output trajectory of the h-closed-loop system tracks the reference signal $p_{d}$ and enters the non-admissible region (the gray, shaded region) as time increases, while that of the s-closed-loop system stays in the admissible region and converges to $\left(p_{1}, p_{2}, v_{1}, v_{2}\right)=(0,0,0,0)$ which is $\Pi_{\mathcal{R}_{s}}\left(\Omega_{h}\right)$.

\section{B. Concave $\mathcal{P}_{a}$}

Consider again the system (20) and the admissible set

$$
\mathcal{P}_{a}=\left\{p=\left[p_{1}, p_{2}\right]^{T} \mid \begin{array}{r}
p_{2} \geq 0, \text { if } p_{1} \geq 0, \\
\text { and } p_{1} \leq 0, \text { if } p_{2} \leq 0
\end{array}\right\} .
$$

Suppose the desired trajectory spans a circle of radius one centered at the origin and given by

$$
p_{d}(t)=[\sin 0.1 t, \cos 0.1 t]^{T} .
$$

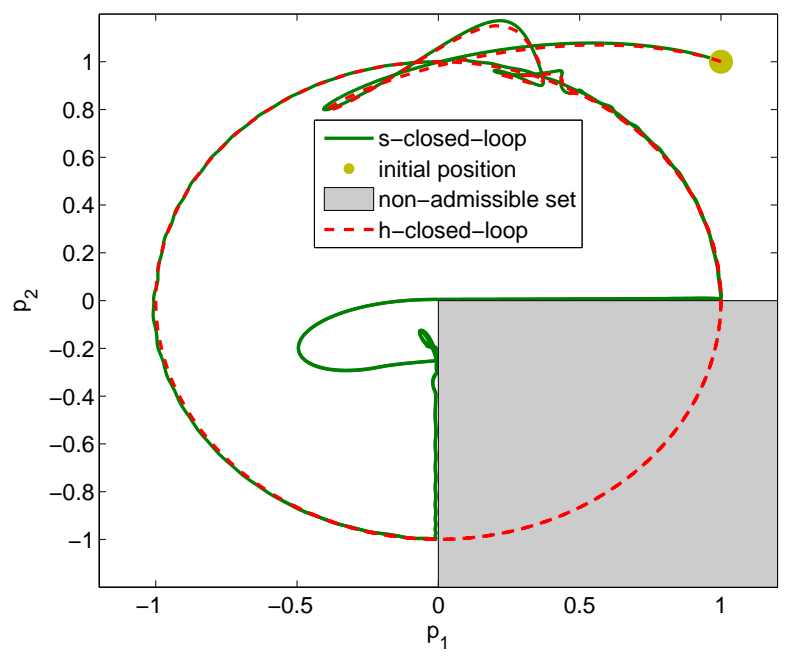

Fig. 2. Trajectories of the system (20) for the set $\mathcal{P}_{a}$ given in (22): $\mathrm{h}$ closed-loop (red, dashed) and s-closed-loop (green, solid). 
Simulation results are displayed in Figure 2 and show the effectiveness of the shared-control algorithm. Note that there is a significant overshoot around the "corner" $\left(p=[0,0]^{T}\right)$ as a consequence of the "discontinuity" caused by the concavity of $\mathcal{P}_{a}$. This is due to the definition of $p_{r}$ in (9) and (11).

\section{CONCLUSIONS}

This paper presents a solution to the output-feedback sharedcontrol problem for fully-actuated, linear, mechanical systems. The algorithm is based on a hysteresis switch to combine the human action $u_{h}$ and the output-feedback control action $u_{o f}$. The theory is developed for convex admissible sets $\mathcal{P}_{a}$ and then illustrated also on nonconvex sets $\mathcal{P}_{a}$. With the shared-control strategy the system configuration is able to reach every point in $\mathcal{P}_{a}$, including boundaries and to remain in the admissible set. Two simple case studies are given in Section IV to illustrate the effectiveness of the s-control design. Future work will focus on the shared-control design for classes of nonlinear systems.

\section{REFERENCES}

[1] S. Nudehi, R. Mukherjee, and M. Ghodoussi, "A shared-control approach to haptic interface design for minimally invasive telesurgical training," IEEE Trans. on Control Systems Technology, vol. 13, no. 4, pp. 588-592, 2005.

[2] Q. Li, W. Chen, and J. Wang, "Dynamic shared control for humanwheelchair cooperation," in Proc. of IEEE Conference on Robotics and Automation, 2011, pp. 4278-4283.

[3] A. Lopes, G. Pires, L. Vaz, and U. Nunes, "Wheelchair navigation assisted by human-machine shared-control and a p300-based brain computer interface," in Proc. of IEEE Conference on Intelligent Robots and Systems, San Francisco, CA, 2011, pp. 2438-2444.

[4] L. Schooley, B. Zeigler, F. Cellier, and F. Wang, "High-autonomy control of space resource processing plants," IEEE Control Systems Magazine, vol. 13, pp. 29-39, 1993.

[5] H. Boessenkool, D. Abbink, C. Heemskerk, F. Helm, and J. Wildenbeest, "A task-specific analysis of the benefit of haptic shared control during telemanipulation," IEEE Trans. on Haptics, vol. 6, no. 1, pp. $2-12,2013$.

[6] M. Mulder and D. Abbink, "Correct and faulty driver support from shared haptic control during evasive maneuvers," in Proc. of IEEE Conference on Systems, Man and Cybernetics, 2011, pp. 1057-1062.

[7] J. Winter and D. Dodou, "Preparing drivers for dangerous situations: A critical reflection on continuous shared control," in IEEE Conference on Systems, Man and Cybernetics, 2011, pp. 1050-1056.

[8] C. Law and Y. Xu, "Shared control for navigation and balance of a dynamically stable robot," in Proc. of IEEE Conference on Robotics and Automation, 2002, pp. 1985-1990 vol.2.

[9] M. O'Malley, A. Gupta, M. Gen, and Y. Li, "Shared control in haptic systems for performance enhancement and training," Journal of Dynamic Systems, Measurement and Control, vol. 128, no. 1, pp. 75-85, 2006.
[10] Y. Li, V. Patoglu, and M. O'Malley, "Negative efficacy of fixed gain error reducing shared control for training in virtual environments," ACM Trans. on Applied Perception, vol. 6, no. 1, 2009.

[11] K. Funabili, T. Iijima, and T. Nojima, "Evaluation of a trajectorybased operation concept for small aircraft: Airborne aspect," in Proc. of Digital Avionics Systems Conference, Indianapolis, USA, 2003, pp. 12.C.5-1-12.C.5-11.

[12] A. Sengstacken, D. DeLaurentis, and M. Akbazadeh-T, "Optimization of shared autonomy vehicle control architectures for swarm operations," IEEE Trans. on Systems, Man and Cybernetics, Part B: Cybernetics, vol. 40, no. 4, pp. 1145-1157, 2010.

[13] A. Lankenau and T. Rofer, "A versatile and safe mobility assistant," IEEE Trans. on Robotics and Automation, vol. 8, no. 1, pp. 29-37, 2001.

[14] K. Goodrich, P. Schutte, F. Flemisch, and R. Williams, "Application of the h-mode, a design and interaction concept for highly automated vehicles, to aircraft," in Proc. of IEEE Conference on Digital Avionics Systems, 2006, pp. 1-13.

[15] H. Chiang, S. Wu, J. Perng, B. Wu, and T. Lee, "The human-in-theloop design approach to the longitudinal automation system for an intelligent vehicle," IEEE Trans. on Systems, Man and Cybernetics, Part A: Systems and Humans, vol. 40, no. 4, pp. 708-720, 2010.

[16] K. Tee, S. Ge, and E. Tay, "Barrier Lyapunov functions for the control of output-constrained nonlinear systems," Automatica, vol. 45, pp. 918-927, 2009.

[17] J. Borenstein and Y. Koren, "The vector field histogram-fast obstacleavoidance for mobile robots," IEEE Journal of Robotics and Automation, vol. 7, no. 3, pp. 278-288, 1991.

[18] Y. Koren and J. Borenstein, "Potential field methods and their inherent limitations for mobile robot navigation," in Proc. of IEEE Conference on Robotics and Automation, 1991, pp. 1398-1404.

[19] J. Lilly, "Evolution of a negative-rule fuzzy obstacle avoidance controller for an autonomous vehicle," IEEE Trans. on Fuzzy Systems, vol. 15, no. 4, pp. 718-728, 2007.

[20] P. Aigner and B. McCarragher, "Modeling and constraining human interactions in shared control utilizing a discrete event framework," IEEE Trans. on Systems, Man and Cybernetics, Part A: Systems and Humans, vol. 30, no. 3, pp. 369-379, 2000.

[21] C. Prieur, "Uniting local and global controllers with robustness to vanishing noise," Math. Control Signals Systems, vol. 14, pp. 143$172,2001$.

[22] L. Zaccarian, "On dynamic control allocation for input-redundant control systems," in Proc. of IEEE Conference on Decision and Control, 2007, pp. 1192-1197.

[23] J. Jiang and A. Astolfi, "Shared-control for fully actuated linear mechanical systems," in Proc. of IEEE Conference on Decision and Control, Florence, Italy, 2013.

[24] C. Prieur and A. Teel, "Uniting local and global output feedback controller," IEEE Trans. Automatic Control, vol. 56, no. 7, pp. 1636$1679,2011$.

[25] B. Chazelle and D. Dobkin, "Optimal convex decompositions," Journal of Computational Geometry, pp. 63-133, 1985. 